\title{
CONOCIMIENTOS, ACTITUDES Y PRACTICAS SOBRE EPILÉPSIA EN ESTUDIANTES DE MEDICINA DEL PRIMER Y SEXTO AÑO
}

\section{Knowledge, Attitudes And Practices About Epilepsy In Students Of Medicine Of The First And Sixth Year ARTÍ́CULO ORIGINAL} \author{
Angel Yaya-Villar ${ }^{1, a}$, \\ 1. Facultad de Medicina, Universidad Nacional San Luis Gonzaga \\ 2. Hospital Regional. Ica, Perú \\ a. Bachiller en Medicina Humana \\ b. Médico especialista en neurología
}

Angel Antonio Anicama-Hernandez ${ }^{2, b}$, Doris Elizabet Huasasquiche-Muñante ${ }^{1, a}$, Fanny Solier-Atiquipa ${ }^{1, a}$, Pedro

\section{Correspondencia:}

Yaya-Villar, Pedro Angel

Domicilio: Urb San Martin de

Porres D 18 - Ica.

Telefono: 947453282

Correo Electrónico:

angel_delcielo88@hotmail.com

Contribuciones De Autoría:

AHAA, HMDE, SAF, YVPA

participaron en el diseño del

estudio, el análisis de los datos,

revisaron críticamente el artículo y

aprobaron la versión final.

Conflicto De Intereses: No

declarados.

Financiamiento: Autofinanciado.

Recibido: 05-11-2017

Aceptado: 20-11-2017

Publicado: 20-12-2017

\section{Como Citar}

Anicama-Hernández A, Huasasquiche-Muñante $D$, Solier-Atiquipa $F$, Yaya-Villar P. conocimientos, actitudes y practicas sobre epilepsia en estudiantes de medicina del primer y sexto año. Rev méd panacea. 2017;6 (3): $98-103$

\begin{abstract}
RESUMEN:
Objetivo: Determinar los conocimientos, actitudes y prácticas sobre epilepsia en los estudiantes del primer y sexto año de la facultad de medicina humana Daniel Alcides Carrión. Ica-Perú, semestre 2017 - I. Material y Metodos: Estudio observacional, descriptivo, transversal en 194 estudiantes de primer y sexto año de la Facultad de Medicina Daniel Alcides Carrión, semestre 2017- I. Se utilizó un cuestionario de 33 preguntas de información demográfica, conocimientos, actitudes y prácticas sobre epilepsia. Se confeccionó la base de datos y análisis en el programa SPSS v. 23, los gráficos y la presentación en Excel y Word 2013. Resultados: Tanto los alumnos de primer y sexto año tienen un conocimiento intermedio con un (41.2\%) y (44.3\%) respectivamente. Además en forma general el $85.5 \%$ del total de los encuestados tienen un conocimiento intermedio. Los alumnos de primer y sexto año tienen una actitud negativa con (26,8\%) y $(25,8 \%)$ respectivamente. Además que en forma general el $52.5 \%$ de los encuestados tienen una actitud negativa hacia la epilepsia, mientras que el $97,9 \%$ tuvo prácticas adecuadas. Se encontró relación entre conocimientos y prácticas, pero no hay relación entre conocimientos y actitudes, de la misma forma que no existe relación entre actitud y práctica. Conclusiones: un alto porcentaje tuvo un conocimiento intermedio, una actitud negativa y una práctica adecuada. Existe relación entre conocimientos y prácticas, mas no entre conocimientos y actitudes ni actitud y práctica.
\end{abstract}

Palabras Claves: conocimientos, actitudes, prácticas, epilepsia (fuente: DeCS BIREME)

\section{ABSTRACT:}

Objetive: To determine the knowledge, attitudes and practices on epilepsy in the first and sixth year students of the human medicine school Daniel Alcides Carrión. Ica-Peru, semester 2017 - I. Material And Methods: Observational, descriptive, cross - sectional study in 194 first and sixth year students of the Daniel Alcides Carrión School of Medicine, semester 2017 -1. A questionnaire was used consisting of 33 questions including demographic information, knowledge, attitudes and Practices on epilepsy. The database and analysis were made in the SPSS v.23, graphics and presentation in Excel and Word 2013. Results: OBoth the first and the sixth year have intermediate knowledge with a (41.2\%) and (44.3\%) respectively. In general, $85.5 \%$ of the respondents have intermediate knowledge. Only 3.6\% of first year students and $1 \%$ of sixth grade students have low knowledge. The first and sixth year students have a negative attitude with (26.8\%) and $(25.8 \%)$ respectively. In addition, in general, $52.5 \%$ of the respondents had a negative attitude towards epilepsy, while 97.9\% had adequate practices. We find an association between knowledge and practices, but there is no association between knowledge and attitudes, just as there is no association between attitude and practice. Conclusions: A high percentage had an intermediate knowledge, a negative attitude and an adequate practice. There is an association between knowledge and practices, but not between knowledge and attitudes or attitude and practice.

Keywords: knowledge, attitudes, practices, epilepsy (source: DeCS BIREME) 


\section{INTRODUCCIÓN}

La epilepsia es uno de los problemas neurológicos más frecuentes a nivel mundial, (1). El término epilepsia, procede del griego "epilambanein" y significa "ser poseído bruscamente por fuerzas exteriores", expresando la concepción animista, y estigmática que desde tiempos inmemoriales asoció a esta enfermedad (2). La Comisión de Epidemiología y Pronóstico de la Liga Internacional contra la Epilepsia define epilepsia como: "Condición caracterizada por crisis epilépticas o convulsiones recurrentes (dos o más) no provocadas por alguna causa inmediatamente identificable. La epilepsia es la afección crónica producida por diferentes etiologías, caracterizada por la repetición de crisis debidas a una descarga excesiva de las neuronas cerebrales (crisis epiléptica o convulsiones) $(3,4)$.

El año 2015 la Organización Mundial de la Salud (OMS), publica que unos 50 millones de personas padecen epilepsia al parecer en todo el mundo, y cerca del $80 \%$ de los pacientes viven en países de ingresos bajos y medianos. (5) Los pacientes y sus familias suelen ser víctimas de la estigmatización y la discriminación en muchas partes del mundo (6).

En Perú el Instituto Nacional de Ciencias Neurológicas (INCN), en el año 2016 encontró que la epilepsia represento la enfermedad más atendida en consulta externa con 2,904 casos, lo que representa el 11.3\%.(7). En Ica, según los archivos de la oficina de estadística de la Dirección regional de salud (DIRESA), en el año 2015 un total de 270 pacientes atendidos con diagnóstico de síndrome convulsivo, siendo el grupo etario con mayor casos, es de 0 a 11 años, con 92 pacientes, siendo el $34 \%$ de la atención total por año de los pacientes con síndrome convulsivo. Se encuentra además que 155 de los casos, fueron de sexo masculino, implicando un porcentaje de $57 \%$.(8)

El estigma social, es una condición, atributo, rasgo o comportamiento que hace que su portador sea incluido en una categoría social hacia cuyos miembros se genera una respuesta negativa y se les ve como culturalmente inaceptable o inferior. (9) La discriminación influye negativamente en la vida de las personas, su entorno familiar y social; se ha descrito en múltiples reportes, que las personas con epilepsia sufren más por la discriminación de la que son objeto que por las crisis. Es importante educar y capacitar a los profesionales de la salud para la eliminación del estigma la cual favorecerá la concientización de la comunidad, posibilitando la mejora de la calidad de vida de los pacientes afectados por epilepsia $(10,11)$. Siendo los profesionales de salud, ya sea neurólogos o médicos generales, los grupos más altamente educados y con mayor influencia en la sociedad, tienen un papel fundamental, influyente y determinante, de cómo la comunidad ve a las personas con epilepsia (12). Motivo por lo que consideramos necesario determinar las actitudes, conocimientos y prácticas de los estudiantes de medicina. De lo cual surge la pregunta ¿Cuáles son los conocimientos, actitudes y prácticas sobre epilepsia en los estudiantes del primer y sexto año de la facultad de medicina humana Daniel Alcides Carrión. Ica-Perú, semestre 2017 - I? Por ello, nos planteamos como objetivo: Determinar los conocimientos, actitudes y prácticas sobre epilepsia en los estudiantes del primer y sexto año de la facultad de medicina humana Daniel Alcides Carrión. Ica-Perú, semestre 2017 - I.

\section{MATERIAL Y MÉTODOS}

El tipo de investigación es Observacional y Transversal. Según el nivel investigación es descriptiva.

Realizado en estudiantes de la Facultad de Medicina Humana Daniel Alcides Carrión de La universidad "San Luis Gonzaga de Ica", durante los meses de febrero y marzo del año 2017, y captados previa autorización de las autoridades pertinentes de la facultad. La población en estudio estuvo constituida por los estudiantes del primer y sexto año de la Facultad de Medicina Humana "Daniel Alcides Carrión" de Ica. Mediante muestreo probabilístico estratificado. Se aplicó la fórmula del muestreo aleatorio simple, calculándose una muestra de 186 estudiantes, para lo que se tomó la estrategia encuestar a número mayor de estudiantes, siendo así que el estudio cuenta el tamaño muestra de 194 estudiantes, cifra válida para nuestro estudio, con un intervalo de confianza al 95\%. Es asi que los participantes cumplieron indefectiblemente con los criterios de inclusión y exclusión.

En cuanto a la Técnicas de recolección y procesamiento de datos, se recolectaron los datos, previa entrevista personal con cada alumno del primer y sexto año de la facultad de medicina humana "Daniel Alcides Carrion", quienes hayan aceptado firmar el consentimiento informado.

Una vez recolectado los datos, se procedió a su análisis en el programa estadístico SPSS Versión 23, para la asociación de variables cualitativas se usó prueba no paramétrica de test de Chi cuadrado de Pearson. Se asumió significación estadística entre las variables para un valor de $\mathrm{p}<0,05$.

Para la presentación de los gráficos y tablas se usaron los programas Excel 2013 y Word 2013.

El instrumento utilizado es una "encuesta", validado por la opinión de expertos. y su fiabilidad fue verificada a través del coeficiente de alfa de Cronbach para conocimiento, actitud y prácticas con valor mayor a 0.75. Constó de 04 partes; la primera con datos sociodemográficos, segunda parte; con preguntas directas para determinar los conocimientos, tercera y cuarta parte por test tipo Likert, para determinar actitudes y practicas sobre epilepsia.

\section{RESULTADOS}

En nuestro trabajo los conocimiento en forma general el $85.5 \%$ del total de los encuestados tienen un conocimiento intermedio, siendo que tanto los alumnos de primer y sexto año tienen un conocimiento intermedio con un $(41.2 \%)$ y $(44.3 \%)$ respectivamente. Además solo el $3.6 \%$ de los alumnos de primer año y el $1 \%$ de los alumnos del sexto año tienen un conocimiento bajo (Figura 1)

\section{Año de estudios}

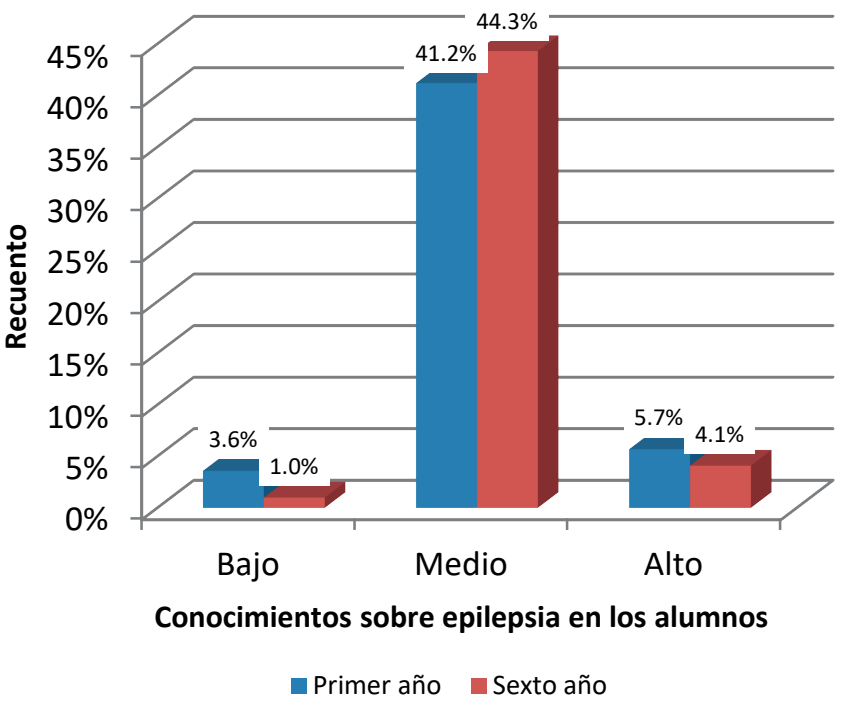

Fuente: Encuesta

Figura 1: Nivel de conocimientos sobre epilepsia en los alumnos de primer y sexto de la facultad de Medicina Humana Daniel Alcides Carrión. Ica Perú Semestre 2017-I

Nuestros participantes consideran que es diferente tener una convulsión que tener una epilepsia (95.9\%). El 88.7\% refieren que la epilepsia no tiene cura. En cuanto a la causa, afirmaron que es una enfermedad del cerebro en un $90 \%$. En cuanto a si el hijo de una persona con epilepsia tendría también esta enfermedad respondieron correctamente en un $78.4 \%$ (Figura 2). 
El hijo de una persona con epilepsia tendría tambien esta...

Factores que desencadenan una convulsión

Una crisis convulsiva puede presentarse como

Por cuanto tiempo deben tomar fármacos antiepilepticos

Con que se debe tratar la epilepsia

Cual es la causa de la epilepsia

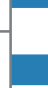

\section{Severidad de la epilepsia}

La epilepsia se cura

Es diferente tener una convulsión que tener epilepsia

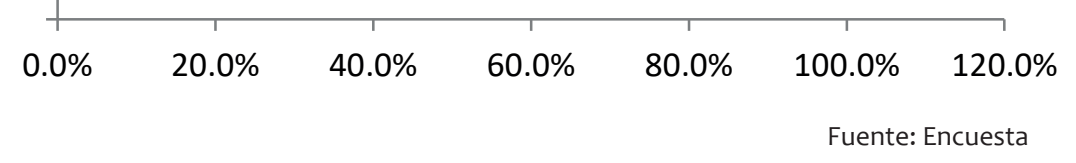

Fuente: Encuesta
$89.2 \%$

$94.3 \%$

$90.0 \%$

$88.1 \%$

$88.7 \%$

Figura 2: Respuestas correctas de conocimientos sobre epilepsia en estudiantes de medicina en estudiantes de primer y sexto año facultad de Medicina Humana. Semestre $2017 \mathrm{I}$.

En cuanto a las actitudes, en forma general el $52.6 \%$ de los encuestados tienen una actitud negativa hacia la epilepsia, siendo que tanto los alumnos de primer y sexto año tienen una actitud negativa con $(26,8 \%)$ y $(25,8 \%)$ respectivamente (Figura 3$)$.

\section{Año de estudios}

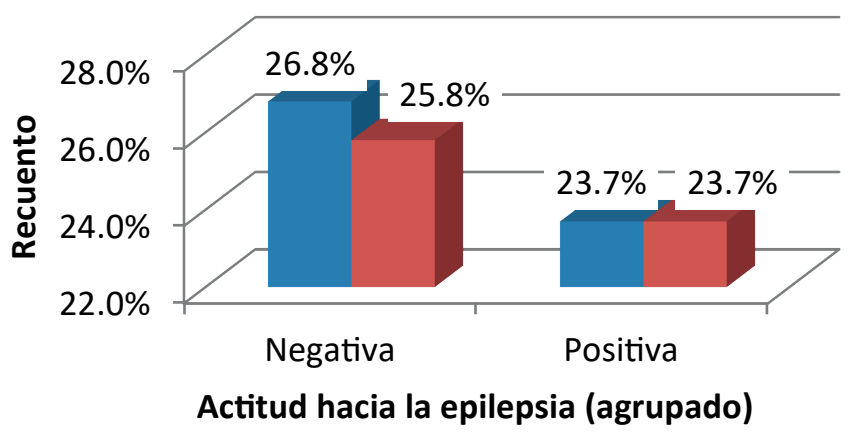

- Primer año $\quad$ Sexto año

Fuente: Encuesta

Figura 3: Actitudes hacia la epilepsia de los alumnos de primer y sexto año de la facultad de Medicina Humana Daniel Alcides Carrión. Semestre 2017-I

Solo el $6.2 \%$ de los estudiantes tiene una actitud positiva al creer que un niño con epilepsia puede asistir al colegio al igual que uno que no padezca de esta enfermedad porque son de igual de inteligentes. El 27,3\% tiene una actitud positiva, al creer que ser un paciente de epilepsia no afecta al desarrollo personal, laboral, educativo y personal. El $21.6 \%$ de estudiantes tienen un actitud positiva, al considerar que un paciente con epilepsia no debería estar en hospital o centro educativo de educación especial. (Fig. 5).
En cuanto a las prácticas en forma general el $97.9 \%$ de los encuestados tienen una práctica adecuada hacia la epilepsia, siendo que tanto los alumnos de primer y sexto año tienen una práctica adecuada (49.5\%) y (48.5\%) respectivamente (Figura 4).

\section{Año de estudios}

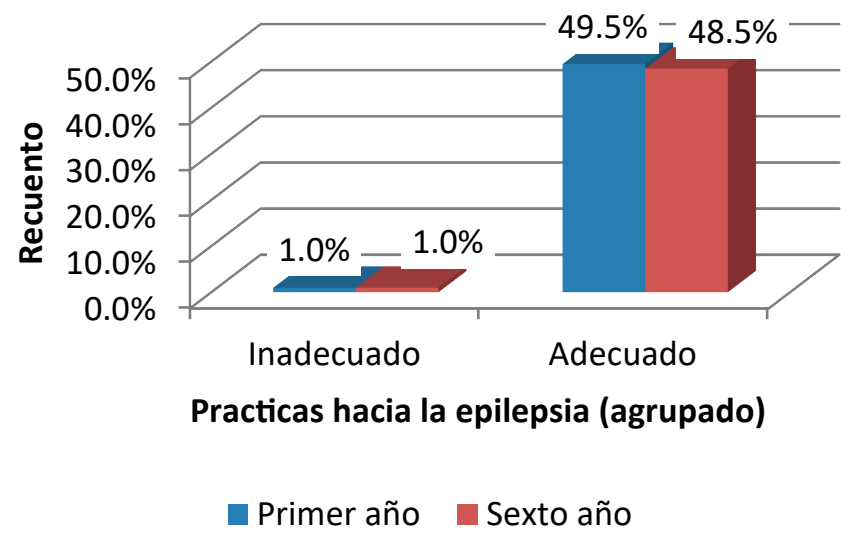

Fuente: Encuesta

Figura 4: Practicas hacia la epilepsia de los alumnos de primer y sexto año de la facultad de Medicina Humana Daniel Alcides carrión. Semestre 2017- I

De los 194 encuestados, el 67\% no introducen objeto en la boca durante una crisis epiléptica, El 91\% de los encuestados mantienen la calma durante una crisis epiléptica. El 95.4\% de los encuestados ponen algo suave debajo de la cabeza. El 94.3\% afloja la corbata, camisa o cualquier cosa del cuello. El $97.9 \%$ se mantiene cerca hasta que la respiración se normalice y comience a levantarse después de una crisis epiléptica. Y el $96.9 \%$ pide ayuda profesional urgente, cuando la persona tarda en recuperarse o el ataque se prolonga más de 5 minutos El $61.9 \%$ de los encuestados no inmovilizan a un paciente cuando tiene una crisis epiléptica (Fig. 6). 
Deben formarse asociaciones de pacientes con epilépsia, para compartir información y experiencias (club de pacientes epilépticos)

El tratamiento del paciente con epilépsia debe ser multidisciplinario (con paoyo psicológico)

Las personas con epilépsia no deberian estar en un hospital o en un centro educativo de educación especial

Ser paciente de epilépsia no afecta el desarrollo personal, laboral, educativo y familiar, por lo tanto deben llevar una

Un niño con epilépsia puede ir a la misma escuela que un niño que no padece de epilépsia porque son igual de inteligentes

Una persona que esta teniendo una crisis epilépticas es incapaz volverse agresiva y atacarlo

No limita el grado de habilidades o desarrollar capacidades en forma permanente. No se considera una discapacidad.

Las crisis epilépticas son esporadicas, las personas con epilépsia son conscientes y capaces el resto de su tiempo

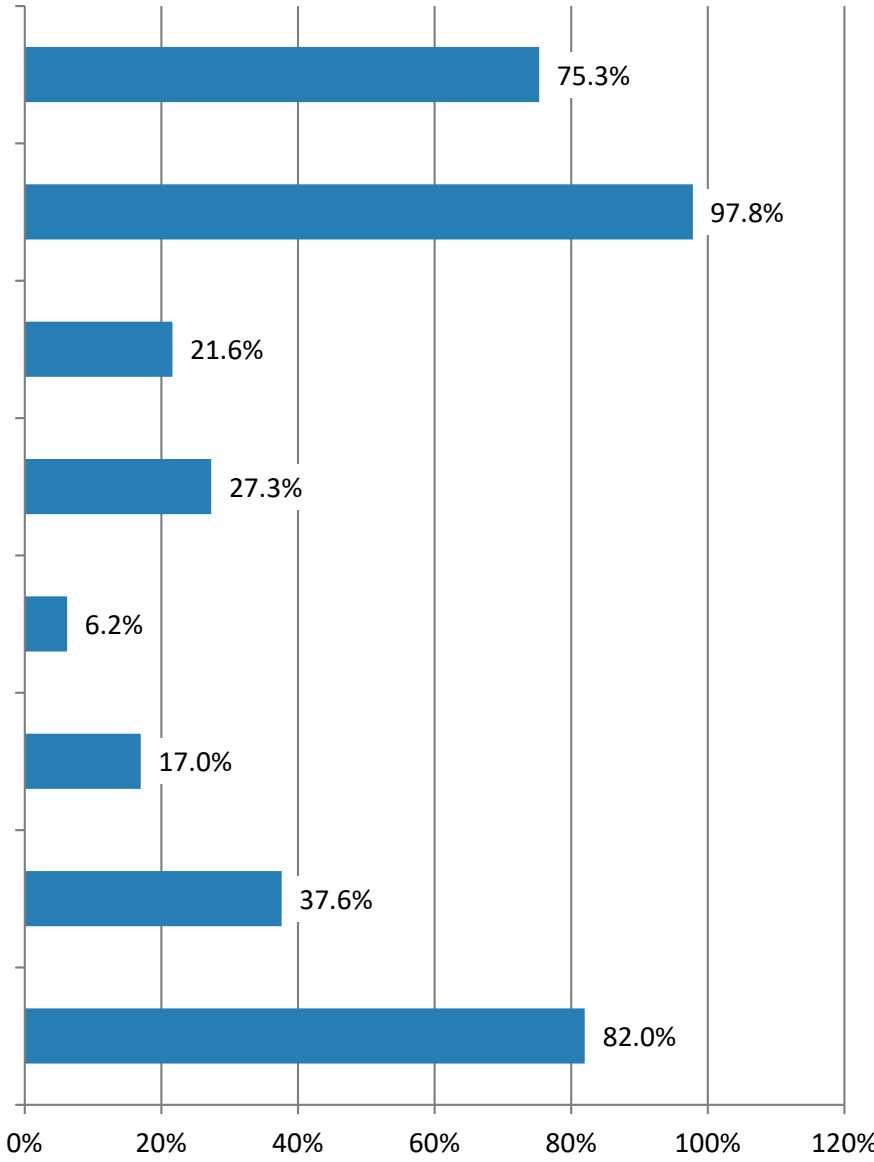

Fuente: Encuesta

Figura 5: Respuestas correctas sobre prácticas sobre epilepsia en estudiantes de medicina en estudiantes de primer y sexto año facultad de Medicina Humana Daniel Alcides Carrión. Semestre 2017 I.

\footnotetext{
Si la persona tarda en recuperarse, el ataque se prolonga más de cinco minutos o le termina uno y comeinza otro, o muestra dificultad en respirar, pido ayuda profesional urgente

Me mantengo cerca hasta que la respiración se normalice y la persona comience a levantarse
}

Coloco el cuerpo del paciente de lado durante una crisis epiléptica

Aflojo la corbata, camisa o cualquier cosa alrededor del cuello

Pongo algo suave debajo de la cabeza

Debo mantener la calma durante una crisis epiléptica.

No debo inmovilizar a una persona que está teniendo una crisis, ya que es muy probable que tenga un reflejo natural, pero voluntario de protegerse.

No debo introducir ningún objeto en la boca cuando alguién tiene una crisis epileptica. Sus consecuencias pueden ser muy perjudiciales.

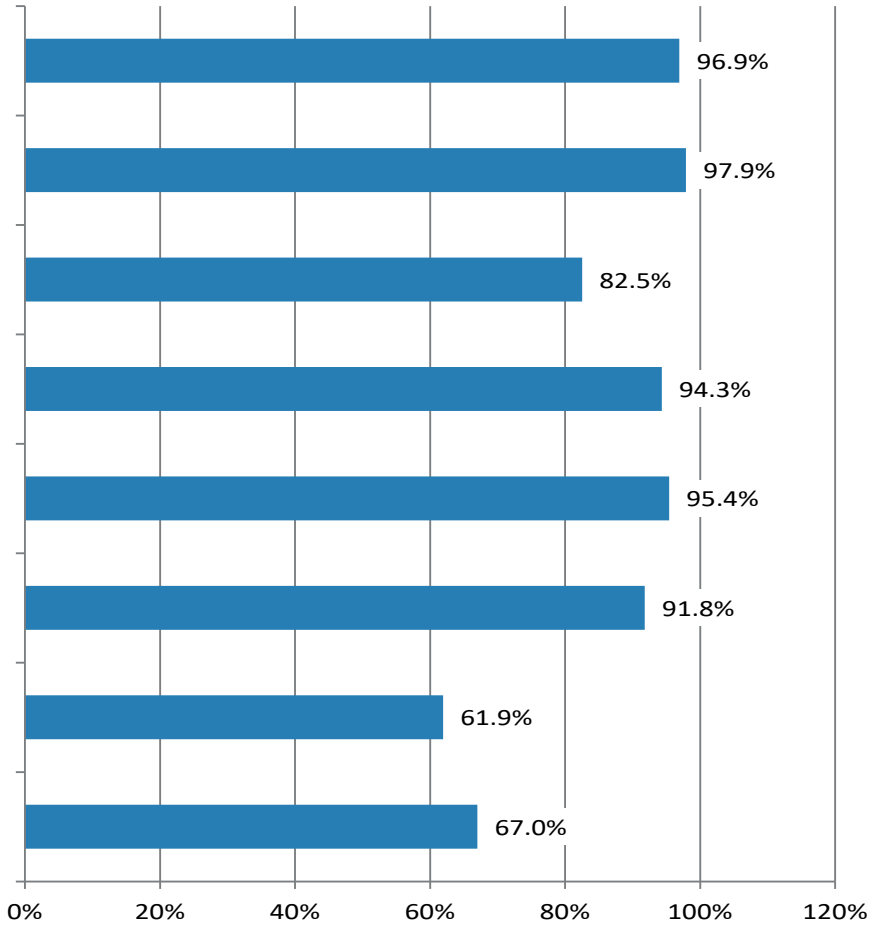

Fuente: Encuesta

Figura 6: Actitudes sobre epilepsia en estudiantes de medicina en estudiantes de primer y sexto año facultad de Medicina Humana Daniel Alcides Carrión. Semestre 2017 I. 
En cuanto a la relación que existe entre los conocimientos y prácticas de nuestros entrevistados, con un Chi cuadrado de Pearson = 0,$00 ; p \leq 0.05$, se determina que si existe asociación entre ambas variables, apreciándose que los que tienen un conocimiento intermedio sobre epilepsia es $85,6 \%$, todos tienen prácticas adecuadas, con ello notamos una relación entre ambas variables.

\section{DISCUSIÓN}

En nuestro trabajo los conocimiento en forma general el $85.5 \%$ del total de los encuestados tienen un conocimiento intermedio, siendo que tanto los alumnos de primer y sexto año tienen un conocimiento intermedio con un $(41.2 \%)$ y $(44.3 \%)$ respectivamente. Además solo el $3.6 \%$ de los alumnos de primer año y el $1 \%$ de los alumnos del sexto año tienen un conocimiento bajo, semejante en el trabajo de Njamnshi y col (13). En el cual el nivel de formación médica del encuestado no parece influir en el nivel de conocimiento de la epilepsia

Nuestros participantes consideran que es diferente tener una convulsión que tener una epilepsia (95.9\%). El 88.7\% refieren que la epilepsia no tiene cura, resultado similar señala Tiamkao y col (14) donde el $71,8 \%$ de los encuestados pensaban que la epilepsia no tenía cura. En cuanto a la causa, afirmaron que es una enfermedad del cerebro en un $90 \%$, contrasta notablemente con Bigelow y col. (15) que solo $45 \%$ de sus encuestados pensaba que la epilepsia era un enfermedad del cerebro.

En cuanto a si el hijo de una persona con epilepsia tendría también esta enfermedad respondieron correctamente en un $78.4 \%$ En su estudio Sudhansu y col. (16) hallo que el $40,3 \%$ consideran que es hereditaria.

En cuanto a las actitudes, en forma general el $52.5 \%$ de los encuestados tienen una actitud negativa hacia la epilepsia, siendo que tanto los alumnos de primer y sexto año tienen una actitud negativa con $(26,8 \%)$ y $(25,8 \%)$ respectivamente.

Solo el $6.2 \%$ de los estudiantes tiene una actitud positiva al creer que un niño con epilepsia puede asistir al colegio al igual que uno que no padezca de esta enfermedad porque son de igual de inteligentes. El los resultados de Bigelow y col. (15) el $55 \%$ de los entrevistados considera que un niño con epilepsia puede tener un alto nivel de inteligencia, y que por lo tanto debe asistir una escuela regular como cualquier otro niño.

Solo el $27,3 \%$ tiene una actitud positiva, al creer que ser un paciente de epilepsia no afecta al desarrollo personal, laboral, educativo y personal; en el estudio de Sudhansu y col. (16) el $77,7 \%$ consideraban que una persona con epilepsia se debe permitir que continúe con su trabajo como cualquier otra persona normal. El $21.6 \%$ de estudiantes tienen un actitud positiva, al considerar que un paciente con epilepsia no debería estar en hospital o centro educativo de educación especial, en el trabajo de Hijazeen y col. (18) el 56,6\% afirma que los niños con epilepsia no deben unirse a las escuelas especiales para personas con discapacidad

En cuanto a las prácticas en forma general el $97.9 \%$ de los encuestados tienen una práctica adecuada hacia la epilepsia, siendo que tanto los alumnos de primer y sexto año tienen una práctica adecuada con (49.5\%) y (48.5\%) respectivamente.

De los 194 encuestados, el 67\% no introducen objeto en la boca durante una crisis epiléptica, ya que pueden ser perjudiciales, semejantes a los estudios de Tiamkao y col (14) y Lineu y col (19) encuentran que, de los encuestados la mayoría recomienda la colocación de una pieza de madera entre los dientes durante una convulsión para evitar que la lengua se mordiera; 50,9\% y $71 \%$ respectivamente, contrasta con en el trabajo de Falavigna y col (20) donde la idea de insertar algo dentro de la boca del paciente durante la convulsión fue mencionada por el 38,8\% de los estudiantes. El $61.9 \%$ de los encuestados no inmovilizan a un paciente cuando tiene una crisis epiléptica, difiriendo notablemente con el trabajo de Lineu y col (19) en el cual solo el $20 \%$ de los encuestados considera que los pacientes deben ser sujetados e inmovilizados durante una crisis convulsiva.
En cuanto a la relación que existe entre los conocimientos y prácticas de nuestros entrevistados, con un Chi cuadrado de Pearson $=0,00 ; p \leq 0.05$, se determina que si existe asociación entre ambas variables, apreciándose que los que tienen un conocimiento intermedio sobre epilepsia es $85,6 \%$, todos tienen prácticas adecuadas, con ello notamos una relación entre ambas variables. En cuanto a la relación que existe entre los conocimientos y actitudes de nuestros entrevistados, con un Chi cuadrado de Pearson= 0,$29 ; p \geq 0.05$, se determina que no existe asociación entre ambas variables, resultado semejante al trabajo de Njamnshi y col (13) en cual un mayor conocimiento sobre epilepsia no afecta a los prejuicios y las actitudes hacia las personas con epilepsia, y a pesar de su buen conocimiento de la epilepsia, las actitudes de los encuestados fueron en general negativas.

En cuanto a la relación que existe entre los actitudes y prácticas de nuestros entrevistados, con un Chi cuadrado de Pearson=0,55; $\mathrm{p} \geq$ 0.05 , se determina que no existe asociación entre ambas variables.

\section{CONCLUSIONES}

Un alto porcentaje tuvo un conocimiento intermedio, una actitud negativa y una práctica adecuada. Existe asociación entre conocimientos y prácticas, mas no entre conocimientos y actitudes ni actitud y práctica. 


\section{BIBLIOGRAFÍA}

1. Burneo J. Epilepsia y manejo de vehículos. Universidad Peruana Cayetano Heredia Lima-Perú, Revista de Neuro-Psiquiatría 2012; 65: 161-165.

2. Fernandes A, Snape b, Beran c, Jacoby. Epilepsy stigma: What do we know and where next?. Epilepsy \& Behavior 22 (2011) 55-6.

3. Mercade J, Sancho R, Mauri LL, Lopez G y Salas P. Sociedad española de neurología. Guías diagnósticas y terapéuticas de la Sociedad Española de Neurología 2012. 1. Madrid. Ediciones SEN.2012.

4. Liga internacional contra la epilepsia. La clasificación etiológica de la epilepsia. (sitio web). Wiley Online Library. disponible en: http://onlinelibrary.wiley.com/doi/10.1111/j.15281167.2011.03041. Acceso 20 marzo del 2015.

5. Organización mundial de la salud "OMS" [sede web]. Epilepsia. Actualizado Mayo del 2015.Centro de prensa. Disponible en: http://www.who.int/mediacentre/factsheets/fs999/es/. acceso 1 de agosto del 2015.

6. Viteva E. Stigmatization of patients with epilepsy: A review of the current problem and assessment of the perceived stigma in Bulgarian patients. Epilepsy \& Behavior 25 (2012) 239-243.

7. Ministerio de Salud del Perú: Instituto Nacional de Ciencias Neurologicas "INCN" [sede web]. Estadísticas 2016: Consulta externa anual2016. Disponible en: http://www.icn.minsa.gob.pe/index.php/menu-estadi/29-esta-2016. acceso 13 de setiembre del 2016.

8. DIRESA ICA. Morbilidad del síndrome convulsivo en la región Ica del 1 de enero al 31 de diciembre del 2015.Oficina de Estadística DIRESA ICA

9. Yang $R$, et al. Stigma of people with epilepsy in china: views of health professionals, teachers, employers, and community leaders. Epilepsy \& behavior. 21 (2011) 261-266.

10. Stucchi P. Estigma, discriminación y concepto de la epilepsia: Rev Neuropsiquiatr 76 (4), 2013.
11. Fandiño J, Franky M. La discriminación y estigmatización a las personas con epilepsia. Sus derechos humanos y civiles ley antidiscriminatoria en Colombia: ISSN medicina (Bogotá) 2011.Vol. 32 No. 2 (89). 160-165.

12. Kestel D, Acevedo C, Medina M, Mesa T. Epilepsia en Latinoamérica: experiencias: Documento técnico basado en las presentaciones del Taller Internacional efectuado en Santiago de Chile, en agosto de 2013, y otras contribuciones posteriores. Santiago de Chile-2013.

13. Njamnshi A, et al. Knowledge, attitudes, and practice of Cameroonian medical students and graduating physicians with respect to epilepsy: Epilepsy \& behavior. 50(2009): 1296-1299.

14. Tiamkao S, et al. Basic knowledge of epilepsy among medical students: J Med Assoc Thai. 2009, 90:2271-2276

15. Bigelow J, Berrett S, Kimuli I, Katabira E. Perceptions of epilepsy among first-year medical students at Mulago Hospital in Kampala, Uganda. Rev Epilepsy Behav. 2015; 51(15):28 - 3.

16. Sudhansu B, et al. Evaluation of knowledge of and attitudes toward epilepsy among the health science students of manipal university: Epilepsy \& behavior 20 (2011) 447-449.

17. Bilguis A, et al. Knowledge of, attitudes toward, and perceptions of epilepsy among university students in Yemen. Epilepsy \& Behavior 52 (2015) 102-107.

18. Hijazeen J, et al. Knowledge, attitudes, and beliefs about epilepsy and their predictors among university students in Jordan. Epilepsy \& Behavior. 41 (2014) 238-243

19. Lineu CF, Rua SS. conhecimentos e atitudes sobre epilepsia entre universitários da área da saúde. Arq Neuropsiquiatra 2009; 62(4):1068-1073.

20. Falavigna A, et al. Awareness and attitudes on epilepsy among undergraduate health care students in Southern Brazil. Journal of Epilepsy and Clinical Neurophysiology. 2009; 15(1):19-23 